\title{
FORCE PARAMETERS AND OPERATING CHARACTERISTICS OF SAFETY-OVERRUNNING BALL-TYPE CLUTCH
}

\author{
Volodymyr MALASHCHENKO ${ }^{1}$, Vladyslav PROTSENKO ${ }^{2}$, Sylwester KLYSZ ${ }^{3,4}$, \\ Valentyn NASTASENKO ${ }^{2}$, Mykhaylo BABIY ${ }^{2}$ \\ ${ }^{1}$ Lviv Polytechnic National University, Lviv, Ukraine, volod.malash@gmail.com \\ ${ }^{2}$ Kherson State Maritime Academy, Kherson, Ukraine, \\ 1904pvo@gmail.com, nastasenko2004@ukr.net, m_babiy@ukr.net \\ ${ }^{3}$ University of Warmia and Mazury, Olsztyn \\ ${ }^{4}$ Air Force Institute of Technology, Warszawa, Poland, \\ sylwesterklysz3@gmail.com
}

\begin{abstract}
Article deals with the field of machinery, namely with the protecting of devices for mechanical driving systems. New construction of safety-overrunning clutch, operating on gearing principle, where safety and overrunning parts are mutually integrated, has been proposed and studied in the article. It has practical importance for building, based on modular principles, machines. As the result of the theoretic studies, the expressions to calculate the following specific clutch torques have been obtained: rating torque, torques of beginning and ending of operation. On the basis of the mentioned studies, the expressions to estimate the clutch main operation characteristics have been obtained - nominal torque exceeding coefficient, accuracy and sensitivity coefficients. These expressions are practically important for proposed clutch calculations. The analysis performed demonstrates that in order to increase the clutch load capacity, the grooves must be made with less inclination angles to clutch axis. Obtained results indicate the advisability of installing the clutch on low-speed shafts. It is shown that the clutch overload sensitivity could be increased by the reduction of the spring rigidity. The calculations performed also demonstrate that balls diameter reduction has a positive effect on the clutch operation parameters. Obtained expressions and numerical results are practically important for proposed clutch calculations. It is shown that in terms of the accuracy of operation, the studied coupling fully corresponds to the level of common and investigated designs of safety clutches.
\end{abstract}

Keywords: safety-overrunning clutch, half-couplings, freewheel clutch, operation accuracy, overload

\section{ACTUALITY AND STATEMENT OF THE PROBLEM}

The problem of creation of effective devices for protection machines from overloads was and still is the pressing problem for machinery science [1]. This problem is additionally complicated with modern tendencies to increase machines' speed and modular building of their elements, which requires creation of dual purpose devices, including safetyoverrunning clutches [2].

\section{ANALYSIS OF RECENT RESEARCH AND PUBLICATIONS}

Now overrunning and safety-overrunning clutches are applied in many types of modern mechanisms, such as transport diesels (mechanical charging driving), automobiles (starters in internal combustion engines, transmissions of electric vehicles) [3-8] metal-cutting machines, robotic systems and prosthesis [9-12], press-lines [13], ball mills $[14,15]$ etc. This devices usually use known operation principles - rollers or special cams wedging between half-couplings surfaces in overrunning clutches and load transfer by friction forces.

Dual purpose clutches creation often goes in the direction of combining two different types of clutches, for example flexible and overrunning, or safety and overrunning in same hull, that became the reason of superposition both their advantages and disadvantages. Reduction of disadvantages number achieves by new operation principle in overrunning clutches - torque transfer through gearing balls and half-couplings grooves, that realized in some successful constructions [16], that confirm their efficiency in long-term operation on automobile starters. Creation of safety-overload clutches, which operating by gearing principle has development reserve through transition from combined constructions [17] to constructions where both parts are mutually integrated, which is reserve to decrease parts number, clutch size, mass and cost.

Taking into account the above, the aim of the article is creation small dimensions safetyoverrunning clutch, working by gearing principle, where safety and overload parts are mutually integrated, and evaluation its basic force parameters 
and operation characteristics, such as accuracy, sensitivity and nominal torque exceeding coefficients $[2,18]$.

In order to achieve this goal, it is necessary to solve the following tasks:

- to propose new construction of ball safetyoverrunning clutch, working by gearing principle, where overrunning and safety parts are mutually integrated;

- to evaluate clutch load capacity, to obtain expressions for its parts loads and torque which clutch able to transmit before safety part operate (rating torque);

- to obtain formulas for minimal and maximal operation beginning torques, and maximal operation process torque in overload mode;

- to evaluate clutch basic operation characteri-stics in overload mode - accuracy sensitivity and nominal torque exceeding coefficients;

- to analyse constructive parameters influence on clutch basic operation characteristics.

\section{STATEMENT OF THE MAIN MATERIAL}

The developed design of a ball-type overrunning safety clutch works as follows. When starting the driving half-coupling 1 (Fig. 1, Fig. 2) clockwise (when looking at the end face A), the balls 6 are moved by the ring 7 which is pressed by the spring 8 along the closed grooves 5 and are inserted into the closed grooves 3 of the driven half-coupling 2 (moving right to left). After the balls 6 are buried in the grooves 3, they will move to the periphery of the grooves 3 and 5 due to the pressure of their oppositely directed side surfaces, and the coupling halves will turn like two threaded parts. After the balls reach the stop in the intermediate ring 12, they stop moving, and, acting on the side grooves 3 of the driven half-coupling 2, they force it to rotate (Fig. 3) with same angular velocity $\omega_{\mathrm{dn}}=\omega_{\mathrm{dg}}$.

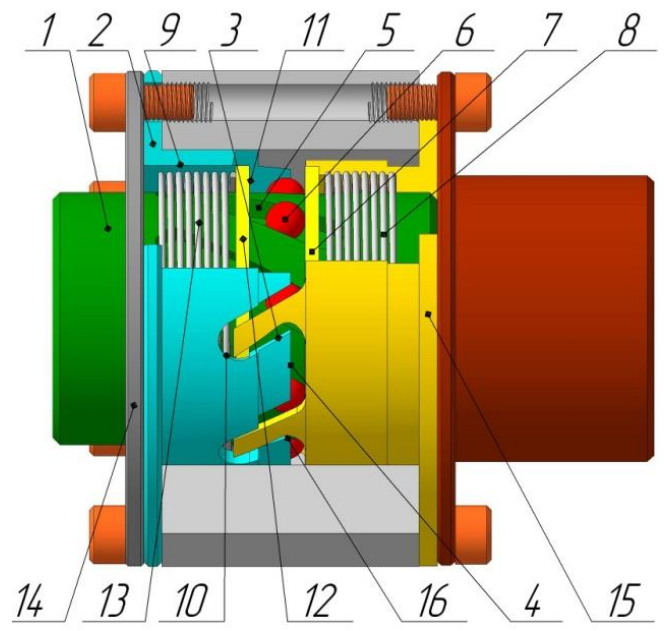

Fig. 1. New overrunning safety clutch scheme

When locking the driving half-coupling 1 , for example, with a brake, or when the engine is turned off, the half-coupling 2 and the masses connected to it will rotate by inertia, while the balls 6 , due to the opposite inclination of the grooves 3 and 5, moving in the grooves 5 (left to right), will be pushed out of grooves 3 due to their opposite inclination, beyond the end 4, compressing spring 8 through ring 7 (Fig. 1, Fig. 2).

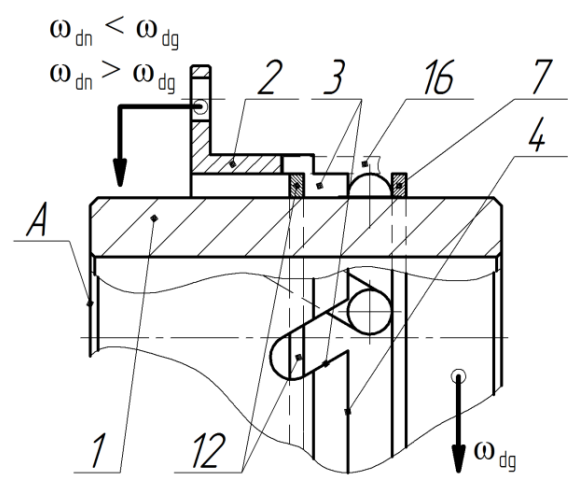

Fig. 2. Clutch operation scheme at the beginning of halfcouplings connection (only main elements are shown)

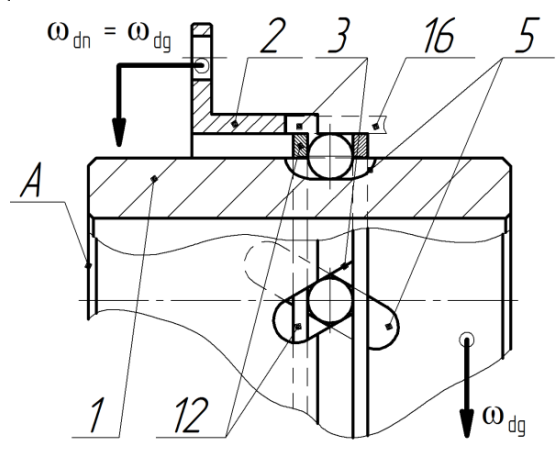

Fig. 3. Clutch operation scheme at the steady mode

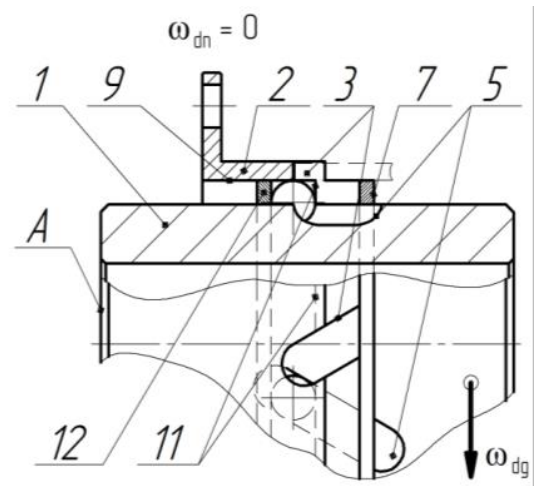

Fig. 4. Clutch operation scheme at the overload mode

Due to this, the coupling halves 2 and 1 will get separated, ensuring the unlocking of the kinematic chain. Similarly, the clutch will work when the direction of rotation of the half-coupling 1 is changed or the angular velocity of the driven halfcoupling 2 exceeds the angular velocity of the driving one $\left(\omega_{d n}>\omega_{d g}\right)$, putting into action its overrunning function. When the clutch is operating in a steady mode (Fig. 3), in case of an increase in the resistance torque on the driven half-coupling 2 (overload mode beginning), the axial load from balls 6 to ring 12 will increase, which will lead to compression of the spring 13 by that (Fig. 4) and subsequent movement of the balls 6 along the 
grooves 5 and 3 inside the hole 9 of the driven halfcoupling 2 (right to left). The hole 9 is blind, bored in such a way that its diameter $D_{1}$ is greater than the diameter $D_{2}$ of the tops of the balls 6 installed in the grooves 5 . When the balls go beyond the end 11 , their contact with the grooves 3 opens and the driving half-coupling 1 starts to rotate freely together with the balls 6 , by unlocking the kinematic chain and eliminating overload - the clutch will put into effect its safety function (Fig. 4).

Bushing 15 (Fig. 1, Fig. 5) serves as a housing for the spring 8 and contains bosses 16 entering in the assembled clutch into open grooves 3 , excluding the extrusion of balls by centrifugal force into the upper part of these grooves when the safety part of the clutch is triggered. In fact, when the clutch is actuated in both directions, the balls 6 move along the inner surface of the bosses 16 , being pressed against it by centrifugal force. Adjustment of the clutch actuation torque is carried out by changing the axial pressure of the spring 13 (Fig. 3) by selecting the thickness of the cover 14 or placing gaskets of the appropriate thickness under it.

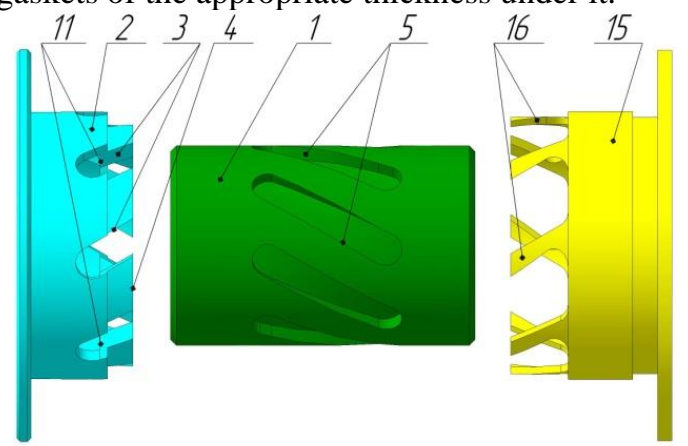

Fig. 5. Clutch main elements construction

In order to determine the clutch load capacity, namely the value of the moment that it is capable of transmitting during the period of steady mode operation, when the balls 6 are in contact with both grooves 3 and 5 and ring 12, mutually stationary during rotation of the clutch (Fig. 3), we compose and analyze the power circuit of the ball installed in the clutch (Fig. 6).

The following calculations have been obtained with several assumptions:

- the angles of inclination to the axis of the oppositely directed grooves of the driving and driven coupling halves are equal in magnitude and equal to $\alpha$;

- the deformations of the elements of the coupling halves are small and do not significantly affect the geometry, and the load between the balls is evenly distributed;

- loads are applied to the balls in the plane of their centers;

- when the clutch is triggered, the balls do not rotate;

- at the beginning of the clutch operation, the force of the spring pressure is equal to the force of its pressure when transmitting the torque rating.
When transmitting the torque rating $T$, each ball is in equilibrium under the action of a system of converging forces (Fig. 6), the condition of which is written as (1):

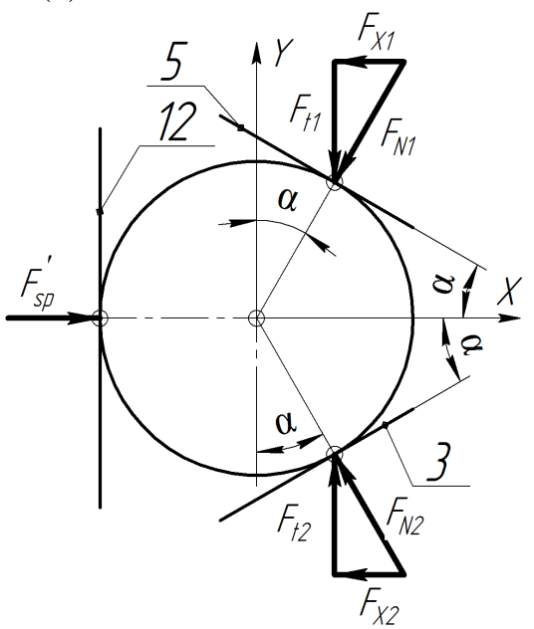

Fig. 6. Ball force scheme at the clutch steady operation mode

$$
F_{N 1}+F_{N 2}+F_{N 3}=0,
$$

where $F_{N 1}$ and $F_{N 2}$ are the pressure rating forces on balls and grooves 3 and 5 side surfaces, which are determined as $F_{N i}=F_{t i} / \cos \alpha$, where $i=1,2$;

$F_{N 3}=F_{s p}^{\prime}$ is the force of normal pressure on the ball from the side of the surface of the ring 12 , equivalent to the force of pressure on this ring of the spring 13 (Fig. 1) from its initial tightening pressure force.

In projections on the $X$ axis:

$$
F_{s p}^{\prime}=F_{X 1}+F_{X 2} .
$$

Considering obtained assumptions and geometric correlations, we can write down:

$$
F_{s p}^{\prime}=2 F_{X i}=2 F_{t} \operatorname{tg} \alpha=\frac{4 T}{z D} \operatorname{tg} \alpha,
$$

where $F_{t}=\frac{2 T}{z D}$ is the tangential force, acting on one ball from the torque $T$;

$D$ is the balls center location circle diameter;

$F_{s p}^{\prime}=\frac{F_{s p}}{z}$ is the part of full spring force, that falls on one ball;

$z$ is the total balls quantity in clutch.

Then torque rating that the clutch is capable of transmitting is determined by the ratio:

$$
T=\frac{D F_{s p}}{4 \operatorname{tg} \alpha} \text {. }
$$

To obtain an expression describing the dependence of the clutch safety part beginning operation torque $T_{b}$ from its parameters, we will consider its elements force interaction in an overload mode. At the beginning of operation (Fig. 7), the balls begin to move relative to grooves 3,5 and ring 12 , sliding along them. 


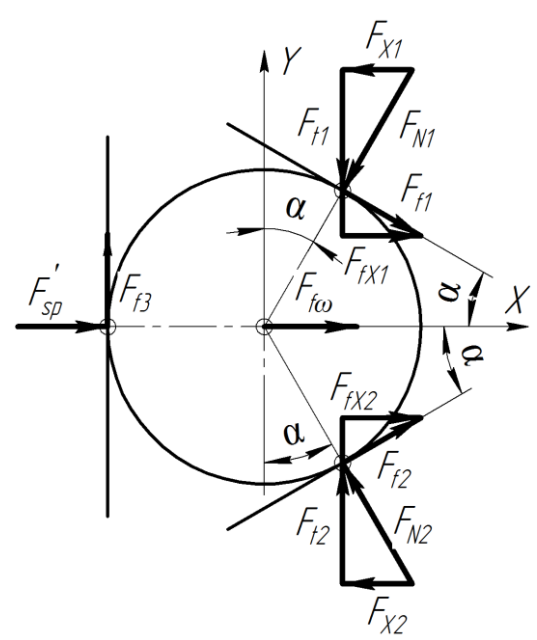

Fig. 7. Ball force scheme at the clutch overload mode beginning

In this case, the condition for their equilibrium in projections onto the $X$ axis will be:

$$
F_{X 1}+F_{X 2}=F_{s p}^{\prime}+F_{f 1 X}+F_{f 2 X}+F_{f \omega},
$$

where $F_{f 1 X}=F_{f 2 X}=F_{N} f \cos \alpha=\frac{2 T}{z D} f$ is the ball friction force on grooves 3 and 5 surfaces;

$F_{f \omega}=m_{b} \omega^{2} D f$ is the ball with mass $m_{b}$ friction force on boss 16 internal surface due to centrifugal force acting;

$\omega$ is the clutch angular velocity;

$f$ is the sliding friction coefficient $(f$-medium, $f_{n}-$ minimum, $f_{m}-$ maximum).

Then, taking into account the above, it is possible to make up the equality:

$$
\frac{4 T_{b}}{{ }_{z} D} \operatorname{tg} \alpha=\frac{4 T}{{ }_{z} D} \operatorname{tg} \alpha+\frac{4 T}{{ }_{z} D} f+m_{b} \omega^{2} D f .
$$

Considering (6), the torque of clutch safety part beginning operation will be:

$$
T_{b}=T+T \frac{f}{\operatorname{tg} \alpha}+\frac{z m_{u} \omega^{2} D^{2}}{4 \operatorname{tg} \alpha} f+T_{f r},
$$

where $T_{f r}=z F_{f 3}=F_{s p} f \frac{D}{2}$ is the torque of balls friction on ring 12 .

Based on the above, expression for safety part operation beginning torque can be presented in forms (8) and (9), which are useful for further

$$
\begin{gathered}
T_{b}=\frac{D F_{s p}}{4 \operatorname{tg} \alpha}\left[1+f \operatorname{ctg} \alpha+\frac{z m_{b} \omega^{2} D}{F_{s p}} f+2 f \operatorname{tg} \alpha\right] ; \\
T_{b}=\frac{D F_{s p}}{4 \operatorname{tg} \alpha}\left[f\left[\operatorname{ctg} \alpha+\frac{z m_{b} \omega^{2} D}{F_{s p}}+2 \operatorname{tg} \alpha\right]+1\right)
\end{gathered}
$$

analyze its operating processes. Half-couplings full disconnect begins (Fig. 8) at the position II, where ball move from position $I$, deforming spring by magnitude $\lambda_{0}$. In position II ball will be on the edge of groove 3 at it intersection with end 11 ( $B$ point).

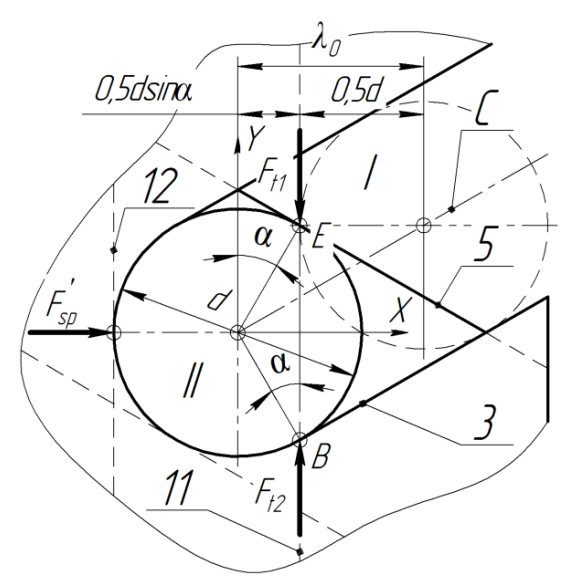

Fig. 8. Ball-groove force interaction scheme at the begin of half-couplings disconnecting in the overload mode (friction forces are not shown conditionally)

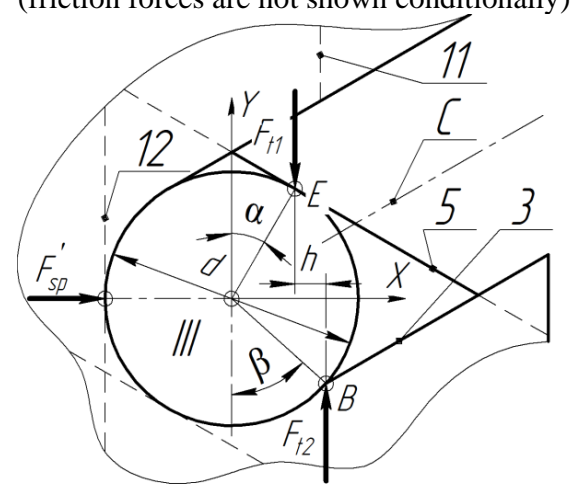

Fig. 9. Ball-groove force interaction scheme at the end of half-couplings disconnecting in the overload mode

Position $I I$ is the last moment when forces $F_{t 2}$ and $F_{t 1}$ acting lines coincide and do not create the forces pair. With further movement of the ball to position $I I I$ (Fig. 9), forces $F_{t 2}$ and $F_{t 1}$ become parallel, and a pair of forces will be created on shoulder $h$ the moment of which will overturn ball around the point $B$ (counterclockwise in Fig. 9), pushing it into the gap between the end 11 and the ring 12.

When the ball (ball diameter - $d$ ) moves from position $I$ to position $I I$, it will move along $X$-axis with magnitude $\lambda_{0}$, and the spring will have equal deformation.

$\lambda_{0}=0.5 d \sin \alpha+0.5 d=0.5(\sin \alpha+1)$.

In this case, the maximum torque of the clutch operation ending (this moment will be the largest transmitted by the clutch to the part of the driving that is protected by it) will be:

$$
\begin{gathered}
T_{\max }=\frac{D\left(F_{s p}+\tilde{N}_{s p} \lambda_{0}\right)}{4 \operatorname{tg} \alpha} \times \\
\times\left(f\left[\tilde{n} \operatorname{tg} \alpha+\frac{z m_{b} \omega^{2} D}{\left(F_{s p}+\tilde{N}_{s p} \lambda_{0}\right)}+2 \operatorname{tg} \alpha\right]+1\right),
\end{gathered}
$$

where $C_{s p}$ - spring rigidity.

Knowing the expressions for the clutch torque rating (4) and torques of it beginning (8) and ending (11) operation, it becomes possible to obtain correlations for estimation the clutch safety part basic operation characteristics, such as: nominal 
torque exceeding coefficient $k_{e}$, accuracy coefficient $\gamma_{a}$ and sensitivity coefficient $\gamma_{s}$

$$
\begin{gathered}
k_{e}=\frac{T_{b}}{T}=1+f \operatorname{ctg} \alpha+\frac{z m_{b} \omega^{2} D}{F_{s p}} f+2 f t g \alpha= \\
=f\left[\operatorname{ctg} \alpha+\frac{z m_{b} \omega^{2} D}{F_{s p}}+2 \operatorname{tg} \alpha\right]+1, \\
\gamma_{a}=\frac{T_{b \max }}{T_{b \min }}= \\
=\frac{f_{m}\left[\tilde{n} \operatorname{tg} \alpha+\frac{z m_{b} \omega^{2} D}{F_{s p}}+2 \operatorname{tg} \alpha\right]+1}{f_{n}\left[\tilde{n} \operatorname{tg} \alpha+\frac{z m_{b} \omega^{2} D}{F_{s p}}+2 \operatorname{tg} \alpha\right]+1} .
\end{gathered}
$$

To illustrate the effect of design and operational parameters on the clutch characteristics when operating in overload mode, calculations for the clutch with following main data have been performed: balls center arrangement diameter $D=58 \mathrm{~mm}$, ball diameter $\mathrm{d}=9.128 \mathrm{~mm}$, balls number $z=8$, grooves inclination angle $\alpha=30^{\circ}$, rotation frequency $n=1500 \mathrm{rpm}$, friction coefficients $f=0.1, f_{m}=0.15, f_{n}=0.05$, spring tightening initial force $F_{s p}=50 \mathrm{~N}$, spring rigidity $C_{s p}=20$ $\mathrm{N} / \mathrm{mm}$. Modeling results are shown on Fig. $10 \div$ Fig. 15.

$$
\begin{aligned}
& \gamma_{s}=\frac{T_{b}}{T_{\text {max }}}= \\
& =\frac{F_{s p}\left(f\left[\operatorname{ctg} \alpha+\frac{z m_{b} \omega^{2} D}{F_{s p}}+2 \operatorname{tg} \alpha\right]+1\right)}{\left[\times\left(f\left[\operatorname{ctg} \alpha+\frac{z m_{b} \omega^{2} D}{\left\{F_{s p}+0,5 C_{s p} d(\sin \alpha+1)\right\}}+2 \operatorname{tg} \alpha\right]+1\right)\right]} .
\end{aligned}
$$

Comparison of the obtained values of the accuracy coefficient $\gamma_{a}$ of the studied clutch with other samples of safety clutches (table 1) demonstrates that in this parameter the new coupling is fully consistent with the level of common designs.

Table 1. Comparison of safety clutches various designs

\begin{tabular}{|l|l|c|}
\multicolumn{1}{c}{ Clutch type } & accuracy coefficients \\
\hline Ball clutch & {$[19]$} & $1.09 \div 1.43$ \\
\hline Ball clutch with reverse switching & {$[20]$} & $1.04 \div 1.28$ \\
\hline Low dynamic ball safety clutch & {$[21]$} & $1.27 \div 1.33$ \\
\hline Planetary safety-flexible clutch & {$[22]$} & $1.05 \div 1.20$ \\
\hline Cam clutch & {$[19]$} & $1.35 \div 2.20$ \\
\hline Two-row cam clutch & {$[23]$} & $1.04 \div 1.28$ \\
\hline Studying clutch & & $1.07 \div 1.50$ \\
\hline
\end{tabular}

\section{DISCUSSION OF RESULTS}

Graphs, shown in Fig. 10 and Fig. 11, illustrate inclination angle $\alpha$ influence on torques (4), (8), (11) (Fig. 10) and coefficients (12), (13), (14) (Fig. 11), which characterize the clutch operation parameters. Their analysis shows that in order to increase the clutch load capacity, the grooves must be made with less inclination angles to clutch axis.
When the $\alpha$ value decreases from $30^{\circ}$ to $10^{\circ}$, the clutch load capacity $T$ increases in 3.25 times (from $1.26 \mathrm{Nm}$ to $4.11 \mathrm{Nm})$. At the same time, the accuracy coefficient $\gamma_{a}$ increases a little (by $15 \%$, from 1.30 to 1.50 ), torque exceeding coefficient $k_{e}$ increases by $23 \%$ (from 1.36 to 1.67 ), clutch sensitivity to overloads increases as well - sensitivity coefficient $\gamma_{s}$ got increased by $18 \%$ (from 0.28 to $0.33)$.

Graphs, shown in Fig. 12 and Fig. 13, demonstrate the dependence accuracy, sensitivity and nominal torque exceeding coefficient of the rotation frequency $n$ and initial spring tightening force $F_{s p}$. When $n$ increases from $100 \mathrm{rpm}$ to $3300 \mathrm{rpm}$, the accuracy coefficient $\gamma_{a}$ increases by $18 \%$ (from 1.25 to 1.48 ), nominal torque exceeding coefficient $k_{e}$ increases by $20 \%$ (from 1.29 to 1.63 ), but the clutch sensitivity to overloads increases $-\gamma_{s}$ by $19 \%$ (from 0.27 to 0.32 ), which indicates the advisability of installing the clutch on low-speed shafts. It is shown that an increase in the spring initial tightening force $F_{s p}$ can significantly increase the sensitivity of the clutch to overloads. Increasing $F_{s p}$ by 2.6 times (from $50 \mathrm{~N}$ to $130 \mathrm{~N}$ ) makes it possible to increase sensitivity coefficient $\gamma_{s}$ by $75 \%$ (from 0.28 to 0.49 ).

Clutch overload sensitivity could also be increased by spring rigidity $C_{s p}$ decreasing (Fig. 14) - changing $C_{s p}$ from $30 \mathrm{~N} / \mathrm{mm}$ to $10 \mathrm{~N} / \mathrm{mm}$ allows sensitivity coefficient to increase more than 2 times (from 0.20 to 0.44 ). The calculations performed also demonstrate (Fig. 15) that balls diameter reduction has a positive effect on the clutch operation parameters.

Reduction of balls diameter by 2 times $(d / D$ from 0.250 to 0.125 ) makes it possible to decrease the accuracy coefficient $\gamma_{a}$ by $13 \%$ (from 1.45 to 1.28 ), nominal torque exceeding coefficient $k_{e}$ by $19 \%$ (from 1.57 to 1.32 ), and to increase sensitivity coefficient $\gamma_{s}$ by $45 \%$ (from 0.22 to 0.32 ).

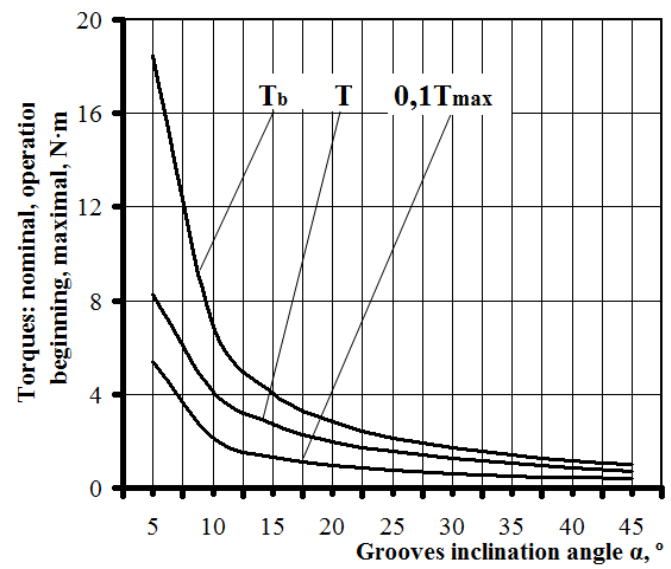

Fig. 10. Influence of the half-couplings grooves inclination angle on the clutch load capacity 


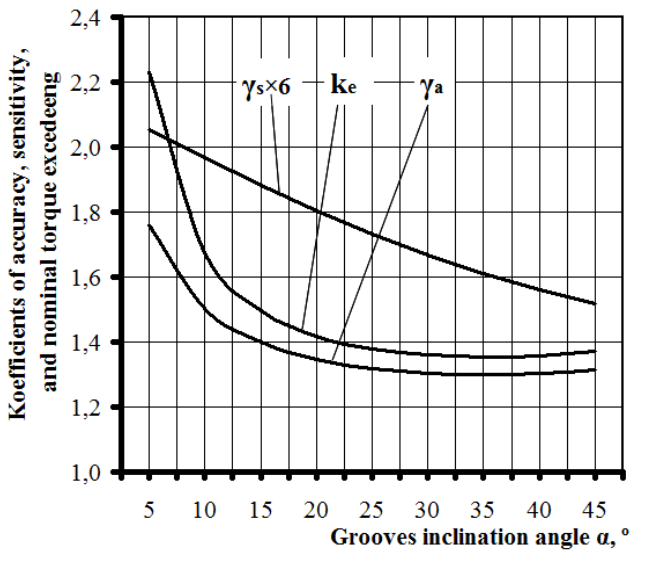

Fig. 11. Influence of the half-couplings grooves inclination angle on the coefficients of accuracy, nominal moment exceeding, and clutch sensitivity

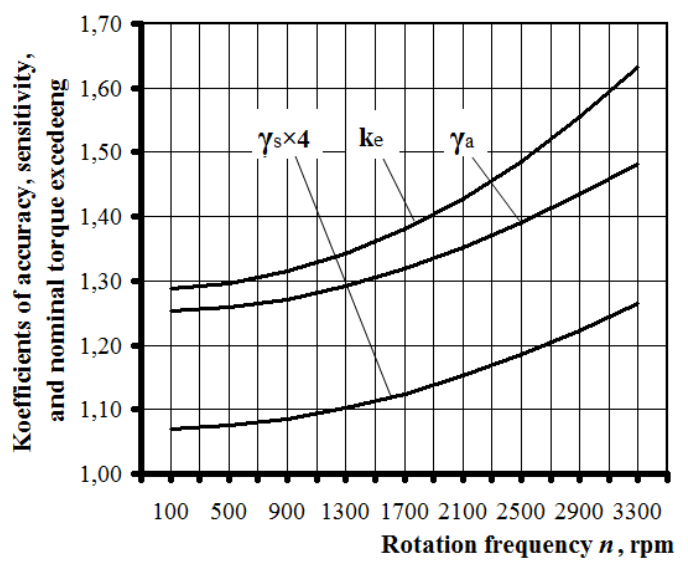

Fig. 12. Influence of the clutch rotation frequency on coefficients of accuracy, nominal moment exceeding, and clutch sensitivity

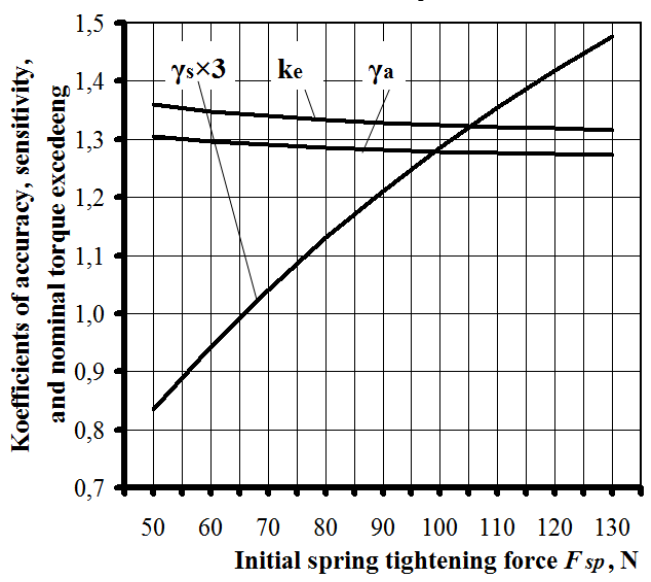

Fig. 13. Influence of the clutch spring initial pressure force on coefficients of accuracy, nominal moment exceeding, and clutch sensitivity

\section{CONCLUSIONS}

1. New construction of safety-overrunning clutch, operating on gearing principle, where safety and overrunning parts are mutually integrated, has been proposed and studied in the article. It has practical importance for building, based on modular principles, machines.

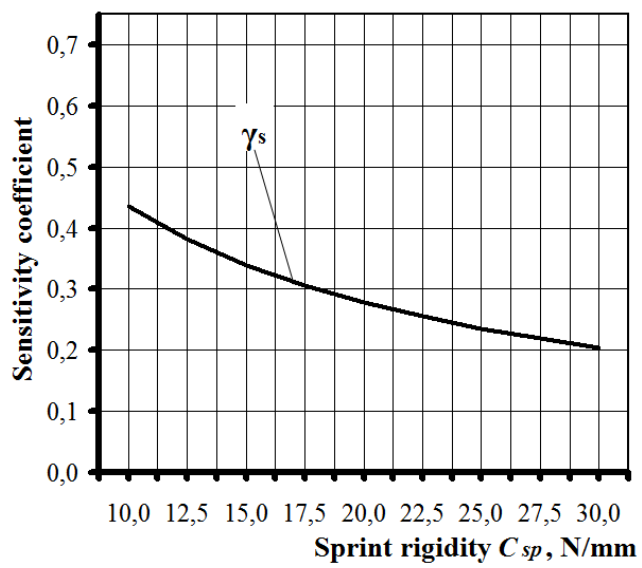

Fig. 14. Influence of the spring rigidity on the clutch accuracy factor

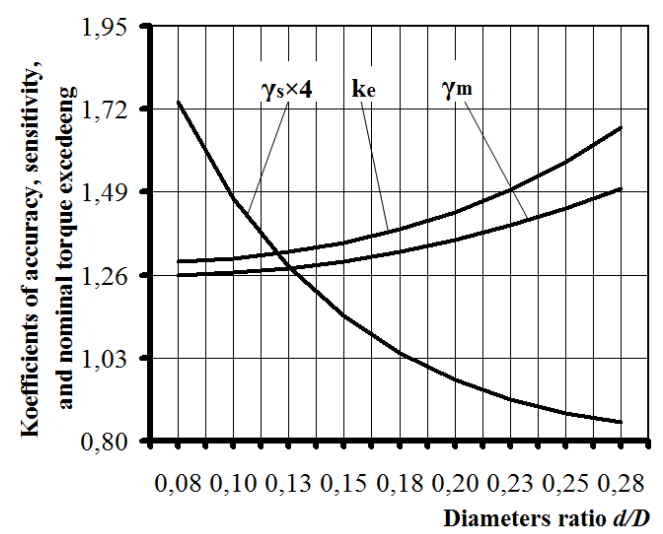

Fig. 15. Influence of balls and half-coupling diameters ratio on factors of accuracy, nominal moment exceeding, and clutch sensitivity

2. As the result of the theoretic studies, the expressions to calculate the following specific clutch torques have been obtained: torque rating, torques of beginning and ending of operation. On the basis of the mentioned studies, the expressions to estimate the clutch main operation characteristics have been obtained - nominal torque exceeding coefficient, accuracy and sensitivity coefficient. These expressions are practically important for proposed clutch calculations.

3. It is shown that in terms of the accuracy of operation, the studied coupling fully consistent to the level of common and investigated designs of safety clutches.

\section{REFERENCES}

1. Malashchenko V, Strilets V, Strilets O, Fedoruk V. New couplings of machine mechanic drivings (in Ukrainian). Publishing house "NUWHP", Rivne, $2019 ; 189$.

2. Hevko B, Lootsiv I, Hevko I, Komar R, Dubynyak T. Flexible-safety clutches: construction, calculation, study (in Ukrainian). Publishing house "FOP Paljanyca V.A. Ternopil, 2019.

3. Nicola F, Sorniotti A, Holdstock T, Viotto F, Bertolotto S. Optimization of a multiple-speed 
transmission for downsizing the motor of a fully electric vehicle. SAE Int. J. Altern. Powertra. 2012; 1 (1): 134-143. https://doi.org/10.4271/2012-01-0630.

4. Hanqi Y, Chenyi Z, Bingzhao G. Fork-less two-speed I-AMT with overrunning clutch for light electric vehicle. Mechanism and Machine Theory. 2018; 130 : 157-169.

https://doi.org/10.1016/j.mechmachtheory.2018.08.019

5. Hyvonen K, Repo P, Lammi M. Light electric vehicles: substitution and future uses. Trans. Res. Proced. 2016;19:258-268. https://doi.org/10.1016/j.trpro.2016.12.085.

6. Roozegar M, Setiawan Y, Angeles J. Design, modeling and estimation of a novel modular multi-speed transmission system for electric vehicles. Mechatronics. 2017;45:119-129.

https://doi.org/10.1016/j.mechatronics.2017.06.002.

7. Roozegar M, Angeles J. The optimal gear-shifting for a multi-speed trans-mission system for electric vehicles. Mech. Mach. Theory. 2017; 116: 1-13. https://doi.org/10.1016/j.mechmachtheory.2017.05.01.

8. Zhou X, Walker P, Zhang N, Zhu B, Ruan J. Numerical and experimental investigation of drag torque in a two-speed dual clutch transmission. Mech. Mach. Theory. 2014;79:46-63.

https://doi.org/10.1016/j.mechmachtheory.2014.04.007.

9. Controzzi M, Bassi Luciani L, Montagnani F. Unified approach to bi-directional non-back drivable roller clutch design. Mechanism and Machine Theory. 2017;116:433-450.

https://doi.org/10.1016/j.mechmachtheory.2017.06.010

10. Convens B, Dong D, Furnmont R, Verstraten T, Cherelle P, Lefeber D. Modeling design and testbench validation of a semi-active propulsive ankle prosthesis with a clutched series elastic actuator. IEEE Robotics and Automation Letters. 2019:1-1. https://doi.org/10.1109/LRA.2019.2897993

11. De Pauw K, Cherelle P, Roelands B, Lefeber D, Meeusen R. The efficacy of the ankle mimicking prosthetic foot prototype 4.0 during walking. Physiological determinants. Prosthetics Orthotics Int. 2018;42:504-510. https://doi.org/10.1177\%2F0309364618767141

12. Hild M, Siedel T, Geppert T. Design of a Passive, Bidirectional Overrunning Clutch for Rotary Joints of Autonomous Robots Intelligent Robotics and Applications. 4th International Conference, Proceedings, Part I. 2011: 397-405.

13. Roczek K, Krol A. Clutch-brake unit - principle of operation and basic diagnostic methods. Diagnostyka. 2018;19(1):33-39. https://doi.org/10.29354/diag/80975.

14. Malashchenko V, Kunytskiy G. Load capacity of large-mass systems (in Ukrainian). Publishing house "Novy Svit-2000", Lviv, 2020; 150.

15. Kharchenko Y, Dragun L. Mathematical modeling of unsteady processes in electrome-chanical system of ring-ball mill. Diagnostyka. 2017; 18(1): 25-35.

16. Malashchenko VO, Hashchuk P, Sorokivsky O, Malashchenko VV. Ball freewheel mechanisms (in Ukrainian). Publishing house "Novy Svit-2000", Lviv, 2012; 212.

17. Boris A. Improvement of the efficiency of mechanical drives by the use of ball fastening and safety couplings (in Ukrainian). Ph.D. dissertation. Lviv Polytechnic National University, Lviv, 2019; 170.

18. Protsenko V. Construction and operation parameters of safety profile clutch on its characteristics influence (in Ukrainan). Technical sciences and technologies. 2017; 1(7): 9-15.

19. Tepinkichiev V. Metal cutting machines safety devises (in Russian). Publishing house "Mashinostroenie", Moscow, 1968; 112.

20. Hevko R. Creation constructions and operation characteristics study of ball safety clutches (in Russian). Ph.D. dissertation thesis. Lviv Polyte-chnic Institute, Lviv, 1991; 17.

21. Buryak M. Justification parameters of low-dynamics ball safety clutches (in Ukrainan). Ph.D. dissertation thesis. Khmelnytsky National University, Khmelnytsky 2004; 20.

22. Pavh I. Justification parameters of parameters separator belt conveyors of beet harvesters (in Ukrainan). Ph.D. dissertation thesis. Lutsk Technical State University, Lutsk, 2000; 20.

23. Matviychuk A. Development and research of doublerow ball and ball-wedge safety couplings of rootharvesting machines (in Ukrainan). Ph.D. dissertation thesis. Ternopil Instrument-making Institute, Ternopil, 1996; 20.

Received 2020-03-07

Accepted 2021-07-06

Available online 2021-08-13

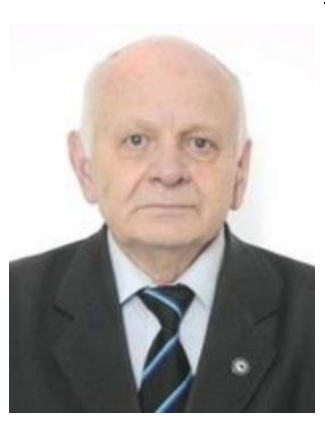

Volodymyr

MALASHSCHENKO.

Doctor of Technical sciences, the professor and the head of the Technical Mechanics and Machines Parts Department of the Lviv Polytechnic National University.

Specializations: calculation of the dynamics of the lifting of high-rise structures; development of new drive clutches.

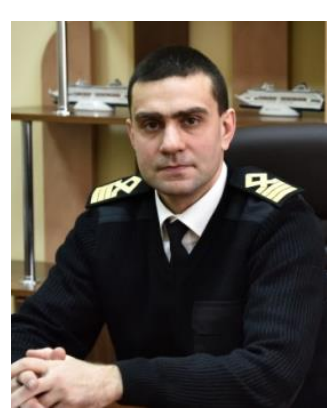

Vladyslav PROTSENKO.

Doctor of Technical sciences, a professor of the Transport Technologies and Mechanical Engineering Department at Kherson State Maritime Academy.

Specializations: development of new driving clutches and overload protection devises.

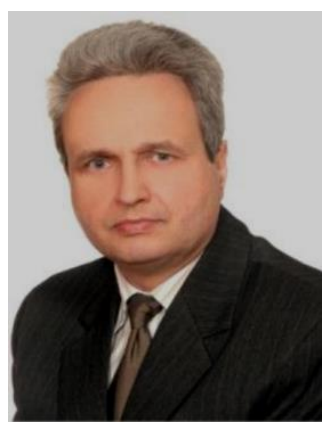

Sylwester KLYSZ.

Professor of Technical sciences, in Airworthiness Division of Air Force Institute of Technology in Warsaw and in Faculty of Technical Sciences at University of Warmia and Mazury in Olsztyn.

Specializations: reliability and durability tests of aviation components and structures, testing of aviation materials. 


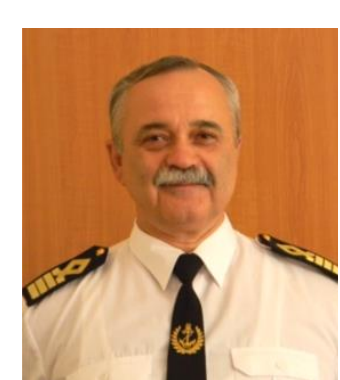

Valentyn NASTASENKO.

Doctor of Technical sciences,

a professor of the Transport

Technologies and Mechanical

Engineering Department at

Kherson State Maritime

Academy.

Specializations: development of new driving clutches and overload protection devises.

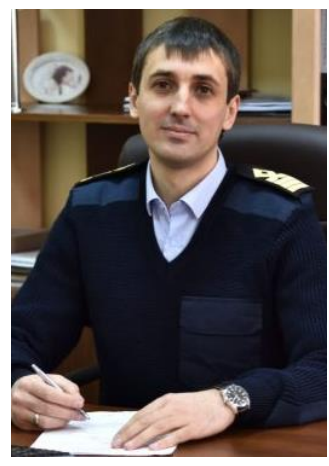

Mykhaylo BABIY.

Ph.D., an associate professor of the Ship Power Plants Operation Department at Kherson State Maritime Academy.

Specializations: development of new driving clutches and overload protection devises. 